\title{
Fatores Associados ao Desperdício de Recursos da Saúde Repassados pela União aos Municípios Auditados pela Controladoria Geral da União*
}

\author{
Factors Associated with the Waste of Health Resources Allocated by the Federal \\ Government to the Municipalities Audited by the Office of the Comptroller General
}

\author{
Lidiane Nazaré da Silva Dias \\ Professora da Faculdade de Ciências Contábeis da Universidade Federal do Pará e Doutoranda do Programa Multi-institucional e Inter-regional de Pós-graduação em Ciências Contábeis \\ da Universidade de Brasília, Universidade Federal da Paraíba e Universidade Federal do Rio Grande do Norte \\ E-mail: lidianedias@ufpa.br \\ José Matias-Pereira \\ Professor Pós-Doutor do Programa Multi-institucional e Inter-regional de Pós-graduação em Ciências Contábeis da Universidade de Brasília, Universidade Federal da Paraíba e \\ Universidade Federal do Rio Grande do Norte \\ E-mail: matias@unb.br
}

Manoel Raimundo Santana Farias

Professor Doutor em Ciências da Área de Controladoria e Contabilidade da Faculdade de Ciências Contábeis da Universidade Federal do Pará

E-mail: manoelfarias@ufpa.br

Vanessa Mayara Souza Pamplona

Mestre do Centro de Registro e Indicadores Acadêmicos da Universidade Federal do Pará e Doutoranda da Universidade Estadual Paulista Julio de Mesquita Filho

E-mail: vanessamayara2@gmail.com

\section{RESUMO}

Este estudo trata dos fatores associados aos desperdícios ativo (corrupção) e passivo (ineficiência) que ocorrem no processo de gestão pública, aqui focado nos gastos com saúde pública. Buscando chamar a atenção para a ocorrência do desperdício passivo, a pesquisa investigou a seguinte questão: quais fatores de desperdício ativo e passivo estão associados com as irregularidades na gestão dos recursos repassados pela União aos municípios brasileiros para a área de saúde no ano de 2010? As evidências empíricas foram obtidas nos relatórios de auditoria da Controladoria Geral da União (CGU) elaborados no ano de 2010 referentes a 102 municípios fiscalizados. Para a identificação do desperdício ativo foram observadas 3 variáveis e para o desperdício passivo 17 variáveis. Os dados relativos às variáveis foram submetidos a uma análise fatorial para agrupá-los em fatores associados estatisticamente com as irregularidades na gestão dos recursos destinados à saúde pública dos municípios auditados. Os resultados da pesquisa não mostraram nível adequado de significância para o desperdício ativo, enquanto que, para o desperdício passivo, evidenciaram três fatores denominados: Fator 1 Inadequabilidade Administrativa (o qual agrupou as variáveis: não pagamento de tributos, não ocorrência de contrapartida e má administração); Fator 2 Fraca Fiscalização (o qual agrupou as variáveis: inexistência ou fraca atuação do conselho e licitação fracionamento); e Fator 3 Baixo nível de Compliance (o qual agrupou as variáveis: licitação irregular e irregularidade não relacionada ao Prefeito). A principal conclusão da pesquisa relaciona-se à identificação da necessidade da implantação de mecanismos de governança no setor público, os quais poderiam reduzir o desperdício ativo e passivo.

Palavras-chave: Gestão de recursos públicos. Desperdício ativo. Desperdício passivo. Análise fatorial. Governança pública.

\section{ABSTRACT}

This study addresses factors associated with active (corruption) and passive (inefficiency) waste that occur in the process of public management and focuses on public health expenditure. Seeking to draw attention to the occurrence of passive waste, this study investigated the following question: what factors of active and passive waste are associated with irregularities in the management of the funds allocated by the Federal Government to Brazilian municipalities for healthcare in 2010? Empirical evidence relating to 102 audited municipalities was obtained from the audit reports of the Office of the Comptroller General (Controladoria Geral da União - CGU), which was established in 2010. Three variables were analyzed for the identification of active waste, and 17 variables were used for passive waste. Data pertaining to the variables were subjected to factor analysis and grouped into factors statistically associated with irregularities in the management of public health funds in the audited municipalities. For active waste, the study results did not show an adequate level of significance, whereas for passive waste, three factors were identified: Factor 1, Administrative Inadequacy, which included the variables "non-payment of taxes", "non-occurrence of matching funds" and "mismanagement"; Factor 2, Weak Supervision, which included the variables "lack of or poor performance of the council" and "fractional bidding" and Factor 3, Low Level of Compliance, which included the variables "irregular bidding" and "irregularities not related to the mayor". The main conclusion of the study is the identification of the need to implement governance mechanisms in the public sector that could reduce active and passive waste.

Keywords: Public funds management, active waste, passive waste, factor analysis, public governance. 
No Brasil, o sistema de saúde foi unificado com a implantação do Sistema Único de Saúde (SUS), em 1988, que deixou de ser responsabilidade de vários ministérios, descentralizou sua gestão e passou a não mais ser exclusiva do Poder Executivo Federal, tornando-se responsabilidade da administração estadual e municipal. Para isto, os Estados e Municípios recebem verbas da União que devem ser aplicadas nos programas e ações de governo. Os dados do SUS são grandiosos, realizando 2,8 bilhões de procedimentos ambulatoriais anuais, 19 mil transplantes, 236 mil cirurgias cardíacas, 9,7 milhões de procedimentos de quimioterapia e radioterapia e 11 milhões de internações (Portal Brasil, 2009).

Certamente estes números são significativos, principalmente se for considerado que o valor pago diretamente pela população atendida é zero, no entanto a saúde pública no país ainda encontra problemas. Frequentemente são noticiadas, em revistas, jornais impressos e televisivos, matérias que denunciam a corrupção ${ }^{1}$, em níveis municipal, estadual, nacional e internacional. $\mathrm{O}$ que não faltam são exemplos antigos e recentes sobre o tema, como o caso que veio à tona, em 2011, referente ao Conjunto Hospitalar de Sorocaba (São Paulo), responsável pelo atendimento de 48 municípios da região. Após uma operação do Ministério Público em conjunto com a Polícia Civil de Sorocaba, 12 pessoas foram presas (entre elas diretores, médicos e dentistas) suspeitas de fraudarem licitações e desviarem recursos públicos da área da saúde (Benites, 2011).

Ao mesmo tempo, como uma doença silenciosa, outro tipo de desperdício de recursos públicos também não pode ser desconsiderado: a ineficiência ${ }^{2}$ da gestão pública (também chamada de má gestão ou, nesta pesquisa, de desperdício passivo), com obras que perduram por meses a mais do que o planejado, remédios comprados e não distribuídos antes do vencimento, ausência de efetiva utilização dos equipamentos recebidos etc. (Controladoria Geral da União, 2011).

Este segundo tipo de desperdício não recebe tanta atenção na mídia ou mesmo em trabalhos acadêmicos como ocorre com a corrupção. Contudo, um estudo recente, realizado por Bandiera, Prat, e Valletti (2009), evidenciou que, na Itália, estes gastos representaram $83 \%$ do total de desperdício de dinheiro público na aquisição de bens.

Tais desvios de recursos em áreas como educação, saúde e saneamento, conforme Ferraz, Finan, e Moreira (2008, p. 2), "[...] geram altos custos para a sociedade já que reduzem a acumulação de capital humano e acentuam a desigualdade porque domicílios mais pobres dependem de maneira mais acentuada dos serviços públicos".

Dentre os serviços públicos prestados à população, é quase consenso que o serviço de saúde é um dos mais importantes, conforme indica um estudo realizado pelo Instituto de Estudos de Saúde Suplementar (IESS) em oito regiões metropolitanas do país, o qual concluiu que, depois da casa própria, o segundo objetivo do brasileiro é dar plano de saúde à família (Rosa, 2011).

A importância da saúde pública é reconhecida pelo Estado, visto ser este o tema de uma das treze Diretrizes do Governo da atual Presidente do Brasil, Dilma Rousseff, contemplada na meta nove "Universalizar a Saúde e garantir a qualidade do atendimento do SUS", que tem como prioridade, de acordo com o Portal Brasil (2011), aperfeiçoar o "Sistema Único de Saúde (SUS) e seus mecanismos de gestão, fiscalização e controle de qualidade aos serviços prestados (...)”.

Em síntese, a saúde é uma das áreas que devem receber maior atenção dos entes governamentais dada a sua importância para o bem-estar da população. Para tanto, faz-se necessária uma gestão financeira adequada dos recursos públicos como um todo, assumindo pressupostos básicos como os princípios da legalidade, impessoalidade, moralidade, publicidade e eficiência, conforme preconiza o artigo 37 da Constituição Federal de 1988.

A ocorrência de desperdícios ativo e passivo de recursos na gestão pública em âmbitos nacional e internacional é um fato evidenciado por Hart, Shleifer, e Vishny (1997), Di Tella e Schargrodsky (2003), Reinikka e Svensson (2005), Ferraz et al. (2008, 2012), Bandiera et al. (2009), Sodré e Alves (2010) e Motta (2010).

Dados sobre tais desperdícios podem ser obtidos nos relatórios da Controladoria Geral da União (CGU), a qual desde 2003 vem realizando fiscalizações in loco nos municípios brasileiros com até 500 mil habitantes, as quais vêm indicando a presença de irregularidades em diversas áreas da administração pública que recebem recursos da União, com destaque, neste trabalho, para a saúde.

Como exposto até o momento, verificam-se diversos problemas na aplicação de recursos na área da saúde, os quais poderiam ser sanados ou ao menos amenizados com uma boa governança pública, como preconizado por Lewis (2006), que afirma que a literatura que trata de governança e corrupção ignora a sua aplicação na área relacionada a políticas públicas sociais, lacuna esta que deve ser preenchida visando à eficiência na utilização destes recursos, pois os gastos adequadamente realizados podem acabar não tendo nenhum impacto se as prioridades não forem cumpridas em virtude de as instituições não funcionarem.

Desta forma, chega-se à seguinte questão de pesquisa: Quais fatores de desperdício ativo e passivo estão associados com as irregularidades na gestão dos recursos repassados pela União aos municípios para a área da saúde no ano de 2010?

Como objetivo, este trabalho pretende identificar os fatores de desperdício ativo e passivo que estão mais associados às irregularidades na gestão dos recursos públicos municipais repassados pela União para a área da saúde.

A principal lacuna a ser tratada no estudo se refere à discussão do tema desperdício passivo, que pode ser o res-

\footnotetext{
A corrupção, chamada de desperdício ativo para fins desta pesquisa, conforme Matias-Pereira (2002, p. 3), "ocorre quando a atividade do funcionário público ou do político não se fundamenta na promoção do interesse geral", ou seja, o ente privado obtém vantagem financeira em detrimento do prejuízo financeiro causado ao ente público.

${ }^{2}$ A ineficiência, chamada de desperdício passivo para fins desta pesquisa, ocorre quando o erário público é lesado, no entanto não há o benefício financeiro do ente privado em contrapartida.
} 
ponsável pela maior parte dos gastos desnecessários de recursos públicos, conforme já comentado.

Assim, esta pesquisa busca contribuir com a gestão e auditoria dos recursos públicos na área da saúde em três níveis: conscientização, prevenção e coibição. Conscientização da sociedade em geral sobre a existência do desperdício passivo, que prejudica a prestação dos serviços públicos e, assim como o desperdício ativo, causa prejuízo ao erário público. Prevenção na medida em que, sabendo claramente quais são os fatores que afetam negativamente os gastos com saúde pública, pode-se criar um programa direcionado de qualificação dos servidores e gestores municipais. Coibição na medida em que pode contribuir com a fiscalização dos recursos destinados aos municípios pelo governo federal para a área da saúde; com a identificação de referidos fatores de desperdício ativo e passivo, a auditoria governamental poderá criar testes específicos visando à identificação destas constatações.

Visando o desencadeamento lógico da proposta, foi elaborada a seguinte estrutura para o artigo: no primeiro tópico tem-se a introdução, com a contextualização, a justificativa, a problemática e o objetivo da pesquisa; no segundo, inicia-se a revisão da literatura; sendo seguida pela apresentação da metodologia, a análise empírica, as conclusões; e, por fim, as referências utilizadas, anexo e apêndice.

\section{REVISÃO DA LITERATURA}

\subsection{O Desafio da Otimização de Recursos.}

Nas entidades privadas, os gastos desnecessários para a elaboração de um bem ou a prestação de um serviço, ou seja, os desperdícios, remetem a um ponto indispensável para a sobrevivência de um negócio: a questão da otimização dos recursos. Em ambientes de acirrada concorrência, as organizações precisam fazer mais com menos para ganhar clientes que se tornam mais exigentes, querendo produtos de alta qualidade a um preço mais baixo.

Exemplo disto vem ocorrendo na área da saúde privada, onde pesquisas têm identificado a profissionalização da gestão hospitalar, que tem passado a adotar ferramentas gerenciais modernas, que já são normalmente utilizadas em outras áreas, buscando com isso unir esforços para o alcance da excelência do atendimento que, por sua vez, gerará satisfação do cliente e, como consequência, aumento no lucro, como no caso da implantação do $\mathrm{Ba}$ lanced Scorecard em hospitais, conforme Carvalho, Dias, e Prochnik (2005).

No entanto, quando se fala em serviços e bens ofertados pelo Estado, em princípio, a questão do desperdício parece não receber tanta importância, talvez pelo fato de o Estado não precisar se preocupar com a concorrência ou mesmo devido ao "valor" pago pelo serviço público pelo cidadão não ser diretamente relacionado ao que foi consumido por ele, sendo esta última uma característica do governo, conforme Niyama e Silva (2011).

Além do mais, contabilidade pública é um tema pouco estudado no Brasil, conforme pesquisas realizadas por Oliveira (2002) e Reis, Slomski, Pereira, e Mello (2007), fazendo com que não contribua tanto quanto poderia para a melhoria da qualidade da informação relacionada ao erário público, que pertence a todos os brasileiros. Assim, a avaliação dos investimentos feitos pelo Estado e o retorno trazido pelos programas desenvolvidos, que têm como seu principal objetivo a contínua melhoria da qualidade de vida da população, podem não ser suficientemente ou eficientemente avaliados.

\subsection{Estrutura Teórica dos Desperdícios Ativo e Passivo conforme Bandiera, Prat, e Valletti (2009).}

De acordo com Bandiera et al. (2009), o desperdício passivo ocorre quando há um gasto desnecessário para o ente público, mas o servidor ou gestor não obtém vantagem financeira para si.

Por sua vez, o desperdício ativo é a corrupção propriamente dita, em que o indivíduo obtém benefício privado, provocando um prejuízo aos cofres públicos, por exemplo, quando ocorre o superfaturamento na contratação de um serviço público (Bandiera, Prat, \& Valletti, 2009).

Com o objetivo de desenvolver uma estrutura teórica para estudar o desperdício ativo e o desperdício passivo, Bandiera et al. (2009) utilizaram como exemplo o trabalho de um gestor de compras de uma entidade pública que deve comprar certa quantidade de produto, pela qual pode pagar um determinado preço. Nesse caso, podem ocorrer duas coisas: a primeira é a possibilidade de o gestor receber benefício direto, "propina", na realização da transação, que eleve o preço e, consequentemente, o gasto público (desperdício ativo); a segunda é a possibilidade de o gestor ser ineficiente e não conseguir otimizar o preço (desperdício passivo).

A questão teórica subjacente é o que leva à ocorrência ou não da primeira possibilidade e também o que ocasiona a eficiência ou ineficiência do gestor prevista na segunda possibilidade.

Uma provável hipótese teórica relacionada à eficiência no gasto público é a seguinte: "O gestor de compras sente uma pressão para manter os preços baixos" (Bandiera et al., 2009, p. 1284). ${ }^{3}$ De acordo com esses autores, isso pode ocorrer em função de uma genuína motivação em maximizar o uso do dinheiro público por ele, o gestor, ter consciência das consequências negativas provocadas pelo pagamento elevado de preços na aquisição de produtos e serviços públicos. Por sua vez, a ineficiência pode ocorrer em função do baixo ou inadequado nível de conhecimento do gestor de compras, ou até mesmo devido à "preguiça", de acordo com Bandiera et al. (2009, p. 1284). 
Uma provável hipótese teórica relacionada ao desperdício ativo, presente quando há recebimento de "propina", é a que segue: "O gestor de compras também pode gostar de benefícios privados" (Bandiera et al., 2009, p. 1284). ${ }^{4}$ Porém, os autores argumentam que a busca por benefícios privados é influenciada pelo risco de o agente sofrer punições. Por exemplo, nas entidades públicas atuantes em ambientes em que esteja instalada uma cultura de denúncia, $o$ risco é maior do que naquelas em que não se tenha o hábito de denunciar os gestores (Bandiera et al., 2009, p. 1284).

Outro aspecto que pode limitar o superfaturamento, este como uma forma de desperdício ativo, é o modo como se exerce a governança corporativa na entidade governamental, na medida em que os mecanismos de governança podem criar restrições ou tornarem menos flexíveis as regras relativas às compras que dificultem o incremento de preços pelos gestores da área (Bandiera et al., 2009, p. 1284).

Em síntese, de acordo com a abordagem teórica anteriormente tratada, os responsáveis pelas compras nas entidades governamentais podem ter mais ou menos custos ou incentivos para lidar com os preços praticados na gestão de compras no âmbito das entidades governamentais.

\subsection{Pesquisas Anteriores.}

A pesquisa desenvolvida por Bandiera et al. (2009), pesquisadores da London School of Economics e Imperial College London, desperta a atenção para o desperdício passivo. Os autores verificaram empiricamente que, na Itália, do total de desperdício que ocorre na aquisição de bens realizada pelo Governo, tal desperdício corresponde a 83\% dos gastos desnecessários, ou seja, é um gasto gerado pela ineficiência na gestão pública.

Outros estudos que tratam do tema foram realizados por Hart et al. (1997), Di Tella e Schargrodsky (2003), Reinikka e Svensson (2005), Ferraz, Finan, e Moreira (2008, 2012), Sodré e Alves (2010) e Motta (2010). Ressalta-se que, conforme Bandiera et al. (2009), a teoria que trata do desperdício passivo é mais recente e menos desenvolvida do que a do desperdício ativo.

Hart et al. (1997, p. 1127 e 1131), pesquisadores da Harvard University e da University of Chicago, levantaram a seguinte questão: "Qual é a eficiência do governo na prestação de serviços públicos?” Tal questionamento é válido a partir do momento em que sua resposta, embasada com dados concretos, consiga respaldar a decisão do gestor público em prestar determinado serviço ou terceirizá-lo para instituições privadas. Com intuito de responder a esta questão, os autores desenvolveram um modelo matemático que analisa a relação custo $\mathrm{x}$ benefício da privatização e o aplicaram às prisões dos Estados Unidos da América (EUA), como pesquisa principal, e, secundariamente, a outros serviços públicos.

A investigação de Hart et al. (1997, p. 1159) apontou:
(...) que a prestação do serviço pelo próprio governo é mais adequada quando a redução dos custos não gera efeito na qualidade, quando inovações de qualidade não são importantes e quando é grave o problema de corrupção nos contratos públicos. Já a privatização do serviço público é mais adequada quando a redução do custo pela redução da qualidade pode ser controlada através de contrato ou competição, quando inovações de qualidade são importantes e quando questões patronais e sindicatos fortes são um problema grave dentro do governo. ${ }^{5}$

Hart et al. (1997) concluíram que, para as prisões, a privatização é mais adequada, ressaltando, no entanto, que nas prisões de segurança máxima, em virtude do elevado índice de violência que faz com que seja necessário o uso da força com maior frequência, não seria adequada a privatização. Nos outros setores em que o modelo foi aplicado verificouse que a terceirização dos serviços públicos nas áreas de política externa, polícia e forças armadas não é adequada; sendo adequada para os serviços de coleta de lixo e produção de armas e, de forma razoável, para as escolas. Para a área da saúde, os autores informam que seria necessário detalhar mais o modelo para que se pudesse concluir algo com mais respaldo.

Seguindo a linha de desperdício de recursos públicos, agora na área de saúde, Di Tella e Schargrodsky (2003), da Harvard University e da Universidad Torcuato Di Tella, realizaram uma pesquisa que verificou que, em 1996 e 1997, houve a redução em aproximadamente $10 \%$ no valor pago por insumos básicos pelos hospitais públicos na cidade de Buenos Aires, Argentina, devido à repressão à corrupção ocorrida no período através de auditorias hospitalares. Paralelamente, os autores estudaram a relação entre o valor dos salários pagos aos funcionários que realizavam as aquisições nos hospitais e a ocorrência de auditorias, concluindo que apenas uma política de melhoria salarial não interfere na questão da eficiência do serviço prestado, assim como apenas uma política ostensiva de auditoria também não resolve o problema, considerando o médio e longo prazo; para que surja efeito, tanto a política salarial quanto a de intensificação das auditorias precisam ser implementadas, de acordo com os autores.

O estudo realizado por Reinikka e Svensson (2005), do World Bank e do Institute for International Economic Studies Stockholm University, buscou identificar quais as formas mais eficazes de aumentar as matrículas e o aprendizado nas escolas primárias. Para isto, realizaram uma pesquisa em Uganda, analisando a estratégia do governo de publicar em jornais dados mensais sobre transferências de recursos públicos para as instituições de ensino, facilitando o acesso e a compreensão da informação pública sobre captação de recursos, e, consequentemente, buscando maior fiscalização. O governo optou por aumentar a transparência ao invés de aumentar as verbas, pois o aumento de recursos não necessariamente resolveria o problema (em virtude de

\footnotetext{
"The purchasing manager may also like private benefits" (Bandiera et al., 2009, p. 1284. Tradução livre).

5 "Our theoretical arguments suggest that the case for in-house provision is generally stronger when noncontractible cost reductions have large deleterious effects on quality, when quality innovations are unimportant, and when corruption in government procurement is a severe problem. In contrast, the case for privatization is stronger when qualityreducing cost reductions can be controlled through contract or competition, when quality innovations are important, and when patronage and powerful unions are a severe problem inside the government." (Hart et al., 1997, p. 1159. Tradução livre).
} 
já ter sido identificado que em muitos países pobres as instituições legais estão entre as mais corruptas). Ao final da análise, os autores identificaram que não só as matrículas e o desempenho escolar haviam melhorado, como também se conseguiu aumentar a captação de recursos para as escolas, evidenciando que o "acesso do público à informação é um poderoso dissuasor para captação de recursos em nível local", conforme Reinikka e Svensson (2004, p. 4) .

Utilizando dados do Brasil, Ferraz et al. (2008, 2012), pesquisadores da Pontifícia Universidade Católica do Rio de Janeiro, da University of California-Berkeley e da Harvard University, estudaram a relação entre a corrupção (desperdício ativo) e má gestão (desperdício passivo) nos resultados da Prova Brasil em português e matemática, concluindo que estes desperdícios, além de reduzirem significativamente os resultados dos estudantes nestas provas, também afetam os indicadores escolares, reduzindo a taxa de aprovação e aumentando a taxa de abandono dos estudantes.

Sodré e Alves (2010), pesquisadores do Massachusetts Institute of Technology, da Universidade de Brasília e da CGU, analisaram os relatórios de auditoria por sorteio público da CGU, buscando verificar se havia relação entre as ementas parlamentares e os indícios de corrupção municipal presentes nos relatórios. Ao final do estudo concluíram que os municípios que obtêm recursos provenientes de tais emendas possuem 25\% mais casos de corrupção, na média. Cabe destacar que os autores também chamam a atenção para a ineficiência da gestão pública municipal que, muitas vezes, pode ocorrer não somente pela falta de capacidade técnica dos servidores ou gestores públicos, mas como algo proposital para tentar "disfarçar" a corrupção.

Ainda no Brasil, pesquisa realizada por Motta (2010), na Universidade Estadual de Campinas, analisou a contribuição do sistema de compras públicas no âmbito da Administração Direta do Poder Executivo, para a maior eficiência do gasto, ou seja, procurando diminuir o desperdício passivo. Motta (2010, p. ix) concluiu que "o sistema brasileiro de compras não contribui substancialmente para a melhora da eficiência do gasto público federal".

\subsection{Contabilidade, Governança e Combate ao Desperdício Ativo e Passivo.}

A contabilidade, em sua atuação na área pública, não deve ser vista apenas como uma obrigatoriedade a ser cumprida ou como um instrumento responsável pelo registro de atos e fatos emanados da administração pública, ela deve ser vista como uma ferramenta capaz de dar suporte, de forma objetiva, à verificação dos atos realizados pelo gestor público e do respeito aos princípios emanados da Consti- tuição Federal, tornando-se um mecanismo de governança ao servir de base para a averiguação da forma como vem sendo utilizado o dinheiro público.

A governança pode ser utilizada como um instrumento de combate à corrupção e à ineficiência no setor público na medida em que ela, segundo Mimicopoulos, Kyj, e Sormani (2007, p. iii), "refere-se aos acordos formais e informais que determinam como as decisões públicas são elaboradas e como as ações públicas são realizadas a partir da perspectiva de manutenção dos valores constitucionais de um país" $\mathrm{Na}$ área da saúde pública, Lewis (2006) afirma que a boa governança acarreta eficácia e eficiência, gerando, inclusive, crescimento econômico. Assim, a governança pública vem ao encontro do objetivo de combate ao desperdício de recursos públicos, podendo proporcionar uma forma de controle dos detentores do poder (Matias-Pereira, 2005).

As auditorias realizadas pela CGU nos municípios se constituem em mecanismos de governança ao serem as responsáveis pela verificação da adequada execução dos programas públicos previamente estabelecidos e da adequada realização dos gastos a eles relacionados. Além disto, também os conselhos municipais de saúde atuam como mecanismos de governança, pois, por meio deles, é garantida a participação do cidadão na elaboração de diretrizes para a política de saúde, acompanhando as ações de saúde, e na fiscalização da utilização dos recursos desta área. Se ambos os mecanismos de governança funcionarem de forma eficaz, poderão levar ao menor nível de desperdício de recursos públicos, pois haveria o aumento do risco de o gestor público ter os atos irregulares descobertos, o que, por consequência, provavelmente aumentaria as chances de punições.

O trabalho da auditoria e da governança são complementares na medida em que fiscalizam a atuação do gestor público, direcionando-o ao atendimento das necessidades da população. Tal importância está expressa na Resolução CFC n. 1.209/09 que trata da comunicação da auditoria com os responsáveis pela governança. Na perspectiva desta resolução, seguindo os bons princípios de governança, os auditores (entendidos como os auditores da CGU para fins desta pesquisa) participariam das reuniões do conselho municipal (entendido nesta pesquisa como um tipo de comitê de auditoria da área pública) de forma regular; o presidente do conselho municipal teria reuniões periódicas com os auditores; e o conselho municipal teria, ao menos uma vez por ano, reunião com o auditor sem a presença da administração (Resolução CFC n. 1209/09). A comunicação entre auditoria da CGU e responsáveis pela governança nos municípios, preconizado na Resolução CFC n. $1209 / 09$, constitui-se em objeto a ser investigado empiricamente dada a sua relevância.

\section{PROCEDIMENTOS METODOLÓGICOS}

Visando o alcance do objetivo geral deste artigo, inicialmente foi realizada uma pesquisa bibliográfica para o levantamento do que vem sendo discutido nacional e in- ternacionalmente sobre o tema, servindo de base também para a classificação das variáveis. Em seguida, para a identificação do desperdício ativo e passivo nos gastos com saúde 
pública municipal, foram utilizados os Relatórios dos Auditores do Programa de Fiscalização por Sorteios Públicos da Controladoria Geral da União (CGU).

A escolha destes relatórios elaborados pela CGU ocorreu em virtude de possuírem como objetivo analisar a aplicação de recursos federais nos municípios, através de visita in loco para a realização de inspeções físicas e documentais, realização de entrevistas, aplicação de questionários e registros fotográficos etc., sendo os Programas de Governo que foram objeto das ações de fiscalização apresentados por Ministério Supervisor (ou seja, é possível identificar a análise específica relacionada aos gastos com saúde do município), discriminando a quantidade de fiscalizações realizadas e os recursos aplicados.

Ressalte-se que, apesar de os relatórios da CGU representarem uma fonte de dados técnica (não científica), eles têm sido utilizados em trabalhos científicos para a construção de base de dados de pesquisas que versam sobre corrupção e/ ou ineficiência, como, por exemplo, Sodré e Alves (2010), Peixoto, Rocha, Nishijima, e Pstali (2012), Avelino, Barberia, e Biderman (2013), bem como a pesquisa desenvolvida por Ferraz e Finan (2007, 2011); e Ferraz et al. (2008, 2012).

A amostra inicial foi composta pelos 120 municípios brasileiros fiscalizados no $31^{\circ} \mathrm{e} 32^{\circ}$ sorteios da CGU, ocorridos no ano de 2010, sendo excluídos os municípios que não apresentaram verbas advindas do Ministério da Saúde, fazendo com que a amostra final fosse de 102 municípios.

As variáveis que classificam o desperdício ativo e passivo utilizadas nesta pesquisa são as mesmas utilizadas por Ferraz et al. (2008), baseada em Ferraz e Finan (2007) ${ }^{8}$, sendo apresentadas a seguir.

Tabela 1 Classificações dos desperdícios ativos e passivos

\begin{tabular}{|c|c|c|c|}
\hline \multicolumn{4}{|r|}{ DESPERDÍCIO ATIVO } \\
\hline $\mathbf{N}^{\circ}$ & Sigla & Variável & Descrição \\
\hline 1 & SF & Superfaturamento & $\begin{array}{l}\text { Ocorre quando os preços pagos estão acima dos preços de mercado, ou quando a quanti- } \\
\text { dade comprada excede em muito as necessidades do município. }\end{array}$ \\
\hline 2 & LIC & Licitação com Desvio de Recursos & Ocorre quando existe evidência de que o serviço licitado e pago não foi prestado. \\
\hline 3 & DR & Desvio de Recursos & Ocorre nos casos em que há despesas sem comprovação da parte física ou serviço prestado. \\
\hline \multicolumn{4}{|r|}{ DESPERDÍCIO PASSIVO } \\
\hline $\mathbf{N}^{\circ}$ & Sigla & Variável & Descrição \\
\hline 1 & NPT & Não pagamento de tributo & Ocorre quando a prefeitura deixa de pagar algum tributo. \\
\hline 2 & NER & Não execução de recurso & Ocorre quando a União repassa o dinheiro, mas o município não o utiliza. \\
\hline 3 & $\mathrm{OI}$ & Obra inacabada & Ocorre quando o prefeito deixa uma obra inacabada e paga a parcela correspondente. \\
\hline 4 & IFAC & Inexistência ou fraca atuação do conselho & Ocorre quando o conselho de saúde não demonstra atuar na fiscalização dos gastos da área. \\
\hline 6 & EIPS & Execução Irregular de programas sociais & Ocorre quando o controle ou o cadastro dos programas sociais encontra-se irregular. \\
\hline 7 & EB & Elefante Branco & $\begin{array}{l}\text { Ocorre quando há uma construção ou compra de bem desproporcional às necessidades } \\
\text { do município. }\end{array}$ \\
\hline 8 & $\mathrm{LI}$ & Licitação Irregular & Ocorre quando existe alguma irregularidade nos documentos da licitação. \\
\hline 9 & LF & Licitação Fracionamento & $\begin{array}{l}\text { Ocorre quando o prefeito fraciona as despesas a serem realizadas em diferentes licitações } \\
\text { objetivando alterar o tipo de licitação necessário, segundo regulamento previsto em lei. }\end{array}$ \\
\hline 10 & LFC & Licitação com Falta de concorrência & Ocorre quando a licitação realizada não apresenta um mínimo de 3 concorrentes. \\
\hline 11 & LL & Licitação com Laranja & Ocorre quando prefeito, na licitação, usa parente ou terceiros em benefício próprio. \\
\hline 12 & DFMS & Desvio de Finalidade para um mesmo setor & $\begin{array}{l}\text { Ocorre quando o recurso é usado também no setor de saúde, mas em objetivo diverso do } \\
\text { que inicialmente acordado com a União. }\end{array}$ \\
\hline 13 & DFOS & Desvio de Finalidade para outro setor & $\begin{array}{l}\text { Ocorre quando o recurso é usado em outro setor, em objetivo diverso do que inicialmente } \\
\text { acordado com a União. }\end{array}$ \\
\hline 14 & $\mathrm{NCP}$ & Não ocorreu contrapartida & Ocorre quando o município não disponibiliza a contrapartida de sua responsabilidade. \\
\hline 16 & INRP & Irregularidade não relacionada ao prefeito & Ocorre quando há irregularidades que não estão sob a responsabilidade do prefeito. \\
\hline 17 & MA & Má Administração & $\begin{array}{l}\text { Qualquer outra irregularidade apontada pela CGU que não se enquadre nos outros crité- } \\
\text { rios acima. }\end{array}$ \\
\hline
\end{tabular}

Fonte: Elaborado a partir das variáveis de Ferraz et al. (2008, p. 13).

Para a elaboração do banco de dados foi criada uma matriz, em excel, que continha em cada linha um dos municípios da amostra e, em cada coluna, uma das variáveis de desperdício acima citadas. Assim, foi realizada a leitura dos relatórios de auditoria da CGU de cada um dos municípios fiscalizados, sendo que a cada irregularidade encontrada referente a determinado município era classificada na coluna a ela correspondente. Ao final somou-se o total de irregularidades encontradas para cada uma das variáveis de desperdício ativo e passivo, em cada município, gerando-se com isto um banco de dados com as constatações de irregularidades (ou "pontos") da área da saúde de cada município pertencente à amostra. No Apêndice 1 são apresentados alguns exemplos de classificação das informações do 
relatório da CGU com base nas variáveis.

Posteriormente, a análise empírica foi realizada a partir da aplicação da técnica estatística multivariada análise fatorial (AF), que foi aplicada com o objetivo de determinar quais desperdícios ativos e passivos estão associados com as irregularidades na gestão dos recursos repassados pela União aos municípios brasileiros para a área de saúde no ano de 2010. A AF é uma técnica multivariada que busca identificar um número relativamente pequeno de fatores comuns que podem ser utilizados para representar relações entre um grande número de variáveis inter-relacionadas (Fávero, Belfiore, Silva, \& Chan, 2009).

A AF parte do pressuposto de que, para a sua realização, os dados provêm de uma distribuição normal multivariada. Porém, Hair, Anderson, Tatham, e Black (2009) afirmam que, apesar de a normalidade univariada não garantir a normalidade multivariada, se todas as variáveis atendem a essa condição, então quaisquer desvios da normalidade multivariada geralmente são inócuos. Assim, para atender este pressuposto foi necessário realizar a transformação de Box-Cox aos dados das variáveis: i) Desperdício Ativo: Licitação com desvio de recursos (LIC) e Desvio de recursos (DR); ii) Desperdício Passivo: Licitação Irregular (LI), Desvio de finalidade para um mesmo setor (DFMS),
Não ocorreu contrapartida (NCP) e Má administração (MA).

Após a verificação da normalidade dos dados, efetuaramse os seguintes procedimentos, de acordo com Fávero, Belfiore, Silva, e Chan (2009) e Field (2009): i) análise da matriz de correlação - esta deve revelar um número substancial de correlações significativas $(p<0,05)$; ii) análise da estatística Kaiser-Meyer-Olkin (KMO) - quanto mais próximo de $1 \mathrm{o}$ seu valor, mais adequada é a utilização da técnica; iii) análise do teste de esfericidade de Bartlett - este teste avalia a hipótese de que a matriz das correlações pode ser a matriz identidade; e iv) análise da Medida de Adequação da Amostra (MAA) - o valor desta medida deve ser maior que 0,50.

O método de obtenção dos fatores foi análise de componentes principais (ACP), utilizado com o objetivo de resumir a maior parte da variância dos dados a um número mínimo de fatores. Para determinar o número de fatores a extrair foi utilizado o critério de Kaiser, que considera todos os fatores com autovalores menores que 1 insignificantes e descartados. Depois de extraídos, os fatores foram rotacionados pelo método ortogonal Varimax.

Para a realização da análise fatorial utilizou-se o software IBM SPSS Statistics, versão 20.

\section{ANÁLISE EMPÍRICA}

Para atender os pressupostos da análise fatorial, inicialmente se procedeu à verificação da normalidade dos dados, sendo que foram necessárias posteriores transformações de Box-Cox para aquelas variáveis que não apresentaram dis- tribuição normal. Após a transformação foi possível verificar que todas as variáveis referentes aos desperdícios ativos e passivos seguem distribuição normal $(p>0,05)$, para um nível de significância de 5\% (Tabela 2).

Tabela 2 Medidas descritivas e teste de normalidade de Kolmogorov-Smirnov (KS) para os dados de desperdício ativo e passivo referentes às irregularidades na gestão dos recursos repassados pela União aos municípios brasileiros para a área de saúde, em 2010

\begin{tabular}{|c|c|c|c|c|c|c|c|c|}
\hline Tipo & Desperdício & Média & Desvio-padrão & Mínimo & Mediana & Máximo & KS & $p$ \\
\hline \multirow{3}{*}{ Ativo } & SF & 0,42 & 0,76 & 0,00 & 0,00 & 3,00 & 0,07 & $>0,15$ \\
\hline & LIC & 0,67 & 1,18 & 0,00 & 0,00 & 6,00 & 0,08 & $>0,09$ \\
\hline & DR & 1,90 & 1,95 & 0,00 & 1,00 & 10,00 & 0,05 & $>0,150$ \\
\hline \multirow{17}{*}{ Passivo } & NPT & 0,09 & 0,45 & 0,00 & 0,00 & 4,00 & 0,06 & $>0,15$ \\
\hline & NER & 0,05 & 0,26 & 0,00 & 0,00 & 2,00 & 0,05 & $>0,15$ \\
\hline & $\mathrm{OI}$ & 0,21 & 0,59 & 0,00 & 0,00 & 4,00 & 0,07 & $>0,15$ \\
\hline & IFAC & 0,46 & 0,56 & 0,00 & 0,00 & 3,00 & 0,08 & $>0,15$ \\
\hline & Client & 0,01 & 0,10 & 0,00 & 0,00 & 1,00 & 0,05 & $>0,15$ \\
\hline & EIPS & 0,64 & 0,95 & 0,00 & 0,00 & 4,00 & 0,09 & 0,09 \\
\hline & $\mathrm{EB}$ & 0,05 & 0,22 & 0,00 & 0,00 & 1,00 & 0,07 & $>0,15$ \\
\hline & $\mathrm{LI}$ & 1,78 & 2,29 & 0,00 & 1,00 & 12,00 & 0,05 & $>0,15$ \\
\hline & LF & 0,13 & 0,39 & 0,00 & 0,00 & 2,00 & 0,08 & $>0,15$ \\
\hline & LFC & 0,18 & 0,43 & 0,00 & 0,00 & 2,00 & 0,09 & $>0,15$ \\
\hline & $\mathrm{LL}$ & 0,02 & 0,14 & 0,00 & 0,00 & 1,00 & 0,05 & $>0,15$ \\
\hline & DFMS & 1,06 & 1,23 & 0,00 & 1,00 & 4,00 & 0,08 & $>0,15$ \\
\hline & DFOS & 0,20 & 0,60 & 0,00 & 0,00 & 4,00 & 0,06 & $>0,15$ \\
\hline & $\mathrm{NCP}$ & 0,85 & 1,10 & 0,00 & 1,00 & 6,00 & 0,08 & $>0,15$ \\
\hline & OAI & 0,13 & 0,44 & 0,00 & 0,00 & 3,00 & 0,08 & $>0,15$ \\
\hline & INRP & 0,02 & 0,14 & 0,00 & 0,00 & 1,00 & 0,06 & $>0,15$ \\
\hline & $M A$ & 9,73 & 5,62 & 1,00 & 8,00 & 30,00 & 0,06 & $>0,15$ \\
\hline
\end{tabular}

Legenda: SF: Superfaturamento, LIC: Licitação com desvio de recursos, DR: Desvio de recursos, NPT: Não Pagamento de Tributo, NER: Não execução de recurso, OI: Obra inacabada, IFAC: Inexistência ou fraca atuação do conselho, Client: Clientelismo, EIPS: Execução irregular de programas sociais, EB: Elefante branco, LI: Licitação irregular, LF: Licitação fracionamento, LFC: Licitação com falta de concorrência, LL: Licitação com laranja, DFMS: Desvio de finalidade para um mesmo setor, DFOS: Desvio de finalidade para outro setor, NCP: Não ocorreu contrapartida, OAI: Obra acabada com irregularidade, INRP: Irregularidade não relacionada ao prefeito, MA: Má administração. 
Posteriormente foi examinada a matriz de correlações nos dados de desperdício ativo, sendo verificado que, de três pares de variáveis, apenas um se apresentou correlacionado (Tabela 3).

Tabela 3 Matriz de correlação para os dados de desperdício ativo

\begin{tabular}{l|c|c}
\hline & SF & LIC \\
\hline LIC & 0,09 & \\
\hline DR & 0,10 & $0,32^{*}$ \\
\hline
\end{tabular}

*significativo: $p<0,05$.

Em relação ao desperdício passivo, verificou-se que as variáveis EIPS, Client, LFC, LL, DFOS, NER, OI, DFMS, EB e OAI não apresentaram um número considerável de correla- ções significativas com as demais variáveis, portanto optou-se por eliminar essas variáveis da análise fatorial (Field, 2009).

A Tabela 4 apresenta a matriz de correlações para as variáveis que permaneceram na análise, correlações significativas foram encontradas entre NPT e NCP, MA. O IFAC mostrou correlações significativas com LF, NCP, INRP e MA. O LI apresentou correlações significativas com LF, INRP e MA. O desperdício LF mostrou correlações significativas com os desperdícios IFAC e NCP. Enquanto que o desperdício NCP apresenta correlações significativas com os desperdícios NPT, IFAC, LF e MA. O desperdício INRP encontra-se correlacionado com os desperdícios IFAC e LI. De modo geral, pode-se observar que o desperdício MA encontra-se correlacionado com todos os outros tipos de desperdícios, exceto com LF e INPR.

Tabela 4 Matriz de correlação para os dados de desperdício passivo referente às irregularidades na gestão dos recursos repassados pela União aos municípios brasileiros para a área de saúde, em 2010

\begin{tabular}{l|l|l|l|l|l|l}
\hline & NPT & IFAC & LI & LF & NCP & INRP \\
\hline IFAC & 0,07 & & & & & \\
\hline LI & 0,10 & 0,07 & & & & \\
\hline LF & 0,05 & $0,27^{*}$ & 0,15 & & & \\
\hline NCP & $0,22^{*}$ & $0,19^{*}$ & 0,03 & $0,19^{*}$ & & \\
\hline INRP & 0,13 & $0,39^{*}$ & $0,24^{*}$ & 0,14 & 0,08 & $0,30^{*}$ \\
\hline MA & $0,29^{*}$ & $0,21^{*}$ & $0,27^{*}$ & 0,11 & 0,14 \\
\hline
\end{tabular}

*significativo: $p<0,05$.

O valor da estatística KMO para o desperdício ativo $(0,54)$ e passivo $(0,61)$ indica a adequação da amostra à análise fatorial. Porém, o nível de significância do teste de esfericidade de Bartlett para o desperdício ativo $(p=0,102)$ indica que a matriz de correlações é igual à matriz identidade, o que mostra que não existem relacionamentos entre as variáveis de desperdício ativo, portanto a análise dos fatores não é apropriada a esses dados. Em relação às variáveis de desperdício passivo, o teste de Bartlett é altamente significativo $(p<0,001)$ e, portanto, a análise dos fatores é apropriada (Tabela 5).

Tabela 5 Teste Kaiser-Meyer-Olkin (KMO) para os dados de desperdício ativo e passivo referentes às irregularidades na gestão dos recursos repassados pela União aos municípios brasileiros para a área de saúde, em 2010

\begin{tabular}{l|l|c|c}
\hline Desperdício & Medida & & Valor \\
\hline \multirow{4}{*}{ Ativo } & KMO & & 0,54 \\
\cline { 2 - 4 } & Teste de Esfericidade de Bartlett & $x^{2}$ & 6,20 \\
\cline { 2 - 4 } & & $p$ & 0,102 \\
\hline \multirow{4}{*}{ Passivo } & KMO & & 0,61 \\
\cline { 2 - 4 } & Teste de Esfericidade de Bartlett & $x^{2}$ & 68,65 \\
\cline { 2 - 4 } & & $p$ & $<0,001$ \\
\hline
\end{tabular}

Os fatores foram retidos pelo critério de Kaiser. A solução fatorial extrai os fatores na ordem de sua importância, assim o Fator 1 explica a maior parcela de variância, com 21,78\%; o Fator 2 explica $21,72 \%$ e o
Fator 3 explica 17,29\%. Desta forma, 60,79\% da variância total são representados pela informação contida na matriz fatorial em termos de três fatores (Tabela 6).

A escolha dos desperdícios que compõem cada um dos fatores ocorre observando-se as cargas fatoriais de cada desperdício, da esquerda para direita e ao longo de cada linha, selecionando-se a carga fatorial com valor maior que 0,50 para fins de interpretação (Field, 2009). Logo, adotando este processo, o Fator 1 tem três cargas significativas, o Fator 2 tem duas e o Fator 3 também tem duas cargas significativas (Tabela 6).

O Fator 1 que agrupou as variáveis não pagamento de tributos, não ocorrência de contrapartida e má administração é chamado de Inadequabilidade Administrativa, sendo o agrupamento de referidas variáveis justificado no fato de ambas representarem negligência na gestão da saúde, pois a verba estava disponível para a utilização e, mesmo assim, a prefeitura deixou de pagar o tributo, de aplicar a contrapartida ou de atender aos fatores necessários à boa gestão.

O Fator 2 composto pelas variáveis inexistência ou fraca atuação do conselho e licitação fracionamento é chamado de Fraca Fiscalização. Com a falta ou fraca atuação do Conselho Municipal de Saúde, as chances de irregularidades relacionadas à gestão ocorrerem passam a ser maiores pois, por exemplo, não é realizado o acompanhamento devido nas aquisições, que podem acabar sendo realizadas de forma fracionada durante $o$ ano. 
O Fator 3, por sua vez, englobou as variáveis licitação irregular e irregularidade não relacionada ao prefeito, sendo denominado de Baixo Nível de Compliance ${ }^{10}$, pois, em ambos os casos, as irregularidades apontadas nas variáveis referem-se ao não atendimento de leis e regulamentos que respaldam o funcionamento e os processos na administração pública.

Os maiores valores de comunalidade foram obtidos para as variáveis LI $(0,72)$, NCP $(0,67)$ e IFAC $(0,67)$, significando que estas variáveis são importantes para explicar a variabilidade total dos dados.

Tabela 6 Matriz de cargas fatoriais após rotação ortogonal pelo método Varimax para os dados de desperdício Passivo referentes às irregularidades na gestão dos recursos repassados pela União aos municípios brasileiros para a área de saúde, em 2010

\begin{tabular}{|c|c|c|c|c|c|}
\hline \multirow[t]{2}{*}{ Desperdício } & \multicolumn{3}{|c|}{ Fator } & \multirow[t]{2}{*}{ Comunalidade } & \multirow[t]{2}{*}{ MAA } \\
\hline & 1 & 2 & 3 & & \\
\hline NPT & 0,72 & $-0,06$ & 0,12 & 0,53 & 0,67 \\
\hline MA & 0,71 & 0,10 & 0,31 & 0,61 & 0,63 \\
\hline NCP & 0,68 & 0,34 & $-0,30$ & 0,67 & 0,66 \\
\hline $\mathrm{LI}$ & 0,18 & 0,01 & 0,83 & 0,72 & 0,53 \\
\hline LF & 0,10 & 0,66 & $-0,04$ & 0,45 & 0,65 \\
\hline IFAC & 0,06 & 0,81 & 0,11 & 0,67 & 0,58 \\
\hline INRP & $-0,02$ & 0,55 & 0,55 & 0,60 & 0,58 \\
\hline Autovalor & 1,52 & 1,52 & 1,21 & & \\
\hline Variância (\%) & 21,78 & 21,72 & 17,29 & & \\
\hline $\begin{array}{l}\text { Variância } \\
\text { Acumulada (\%) }\end{array}$ & 21,78 & 43,50 & 60,79 & & \\
\hline
\end{tabular}

\section{CONCLUSÕES}

Esta pesquisa foi estruturada objetivando identificar os fatores de desperdício ativo e passivo mais associados com as irregularidades na gestão dos recursos públicos municipais repassados pela União para a área da saúde.

Para o alcance deste objetivo, realizou-se uma pesquisa teórico-empírica. Na primeira parte foi identificada a base teórica para a realização do estudo. Na parte empírica, dado o objetivo da pesquisa, utilizou-se a técnica multivariada de análise fatorial.

O resultado da pesquisa apontou que, para o desperdício ativo, não é possível, com base nos dados analisados, apresentar um fator que melhor o defina, pois o resultado do teste de Bartlett não foi significativo. Uma provável causa para o ocorrido é o fato de terem sido utilizadas apenas três variáveis relacionadas a ele. Quanto ao desperdício passivo, foram encontrados três fatores que mais se associaram com as irregularidades ora em estudo.

O Fator 1 foi chamado de Inadequabilidade Administrativa, tendo agrupado as variáveis "não pagamento de tributos", "não ocorrência de contrapartida" e "má administração". Este fator vem ao encontro do que foi evidenciado por Bandiera et al. (2009) na literatura do desperdício passivo, os quais identificaram que há gestores públicos ineficientes e que não conseguem otimizar os recursos, fato que pode ocorrer devido a não possuírem o conhecimento adequado a realização da função ou por serem "preguiçosos". Uma consequência prática deste problema ocorre quando o Governo Federal deixa de repassar a verba referente a algum programa a ser desenvolvido no município em virtude de este último não ter dado a contrapartida necessária (a qual, algumas vezes, representa um valor percentual muito pequeno quando comparado ao montante do valor do programa); ressalta-se que há casos em que o município possui a verba disponível e mesmo assim não faz a contrapartida previamente acordada.

O Fator 2 foi chamado de Fraca Fiscalização, sendo composto pelas variáveis "inexistência ou fraca atuação do conselho" e "licitação fracionamento". Um ponto a ser esclarecido é que o Conselho Municipal de Saúde é fundamental para a eficiência na prestação do serviço público na saúde devido a sua atuação na formulação de estratégias, no controle da execução da política e na fiscalização econômico-financeira da área, o que, dentre outros benefícios, inibiria a ocorrência de licitação fracionamento. Este Fator, Fraca Fiscalização, pode levar à ocorrência do desperdício ativo, conforme apresentado na literatura por Bandiera et al. (2009), visto que servidores e gestores que possuem tendência a buscar benefícios privados o fazem proporcionalmente ao risco de sofrer punições. $\mathrm{O}$ combate a este Fator por meio da efetiva atuação do Conselho poderia reduzir o desperdício passivo, de acordo com o identificado na pesquisa de Di Tella e Schargrodsky (2003).

Por fim, o Fator 3 que foi chamado de Baixo Nível de Compliance, englobando as variáveis "licitação irregular" e "irregularidade não relacionada ao prefeito". Este Fator relaciona-se com o não atendimento de regras, normas e diretrizes previamente estabelecidas, que poderia ser trabalhado com a implantação de mecanismos de governança adequados, fazendo com que os servidores e gestores fossem pressionados a serem eficientes, conforme proposto por Bandiera et al. (2009).

A principal contribuição desta pesquisa refere-se à necessidade de se chamar a atenção da academia, do governo e da sociedade em geral para a existência do desperdício passivo, que afeta a qualidade dos serviços prestados à população 
e provoca prejuízo aos cofres públicos, podendo consumir uma quantia até 4 vezes maior do que o desperdício ativo, como no caso italiano identificado por Bandiera et al. (2009). Não obstante, o desperdício passivo pode ser uma forma de esconder o desperdício ativo, como apresentado por Sodré e Alves (2010). Como consequência prática da pesquisa foram identificados argumentos teórico-empíricos que sugerem que o desperdício passivo pode ser reduzido por meio de uma série de ações, tais como: (i) aumento das fiscalizações; (ii) uma maior transparência das informações relacionadas aos programas de governo (funcionamento e execução) de forma a tornar a informação do setor público efetivamente acessível e compreensível ao cidadão; (iii) implantação de programas de capacitação e motivação do servidor e gestor público, fazendo com que internalizem a importância de sua adequada atuação profissional, buscando também, paralelamente, criar uma cultura de denúncia de irregularidades praticadas no serviço público; (iv) realização de uma análise verificando se determinado serviço deve ser mesmo presta- do pelo Estado ou terceirizado; e (v) implantação de novos mecanismos de governança e fortalecimento dos já existentes, como a auditoria da CGU, os quais poderiam reduzir o desperdício ativo e passivo.

Ressalta-se que, apesar de os relatórios de auditoria da CGU apresentarem constatações que podem ser questionadas pelos prefeitos, o fato de haver o "ponto" no relatório já é evidência da ocorrência de determinado desperdício, visto o relatório estar respaldado em documentos ou falta de documentos que justifiquem os atos realizados pelos servidores e gestores municipais. No entanto, pode-se apontar esta como sendo uma limitação da pesquisa.

Para pesquisas futuras sugere-se: (i) a utilização de uma base de dados maior na área da saúde (nesta foram utilizados os relatórios de auditoria da CGU do ano de 2010, referentes aos sorteios $31^{\circ}$ e $32^{\circ}$ ); (ii) a ampliação das variáveis de identificação do desperdício ativo; e (iii) a ampliação da pesquisa para outras áreas da gestão municipal que recebem recursos da União, como a área da educação, por exemplo.

\section{Referências}

Avelino, G., Barberia, L. G., \& Biderman, C. (2013, February). Governance in managing public health resources in Brazilian municipalities. Health Policy and Plan. First published online, 1-9.

Bandiera, O., Prat, A., \& Valletti, T. (2009). Active and passive waste in government spending: evidence from a policy experiment. American Economic Review, 99 (4), 1278-1308.

Benites, A. (2011, Junho). Após prisões, governo intervém em hospital de Sorocaba. Folha.com. São Paulo. Recuperado em 10 agosto, 2011, de http://wwwl.folha.uol.com.br/cotidiano/931182-apos-prisoesgoverno-intervem-em-hospital-de-sorocaba.shtml.

Carvalho, F. de M., Dias, L. N. da S., \& Prochnik, V. (2005, novembro). A utilização do Balanced Scorecard em hospitais: o caso do Hospital Nove de Julho. Anais do Congresso Internacional de Custos, Florianópolis, SC, Brasil, 9.

Constituição da República Federativa do Brasil de 1988. (1988). Recuperado em 8 julho, 2012, de http://www.senado.gov.br/legislacao/const/ con1988/CON1988_29.03.2012/CON1988.pdf.

Controladoria Geral da União. CGU. (2011). Relatório de fiscalização. Recuperado em 10 agosto, 2011, de http://www.cgu.gov.br/sorteios/ indexl.asp.

Di Tella, R., \& Schargrodsky, E. (2003). The role of wages and auditing during a crackdown on corruption in the city of Buenos Aires. Journal of Law and Economics, 46 (1), 269-92.

Fávero, L. P., Belfiore, P., Silva, P., \& Chan, B. (2009). Análise de dados: modelagem multivariada para tomada de decisões. Rio de Janeiro: Campos Elsevier.

Ferraz, C., \& Finan, F. (2007, June). Electoral accountability and corruption in local governments: evidence from audit reports. Germany: Institute for the Study of Labor, IZA, IZA Discussion Paper 2843.

Ferraz, C., \& Finan, F. (2011, June). Electoral accountability and corruption: evidence from the audits of local governments. American Economic Review, 101 (4), 1274-1311.

Ferraz, C., Finan, F., \& Moreira, D. B. (2008). Corrupção, má gestão, e desempenho educacional: evidências a partir da fiscalização dos municípios. Anais do Encontro Nacional de Economia, Salvador, BA, Brasil, 36.

Ferraz, C., Finan, F., \& Moreira, D. B. (2012, May). Corrupting learning: evidence from missing federal education funds in Brazil. Forthcoming. Journal of Public Economics, 96, 712-726.

Field, A. (2009). Descobrindo a estatística usando o SPSS. (2. ed.). Porto Alegre: Artmed.

Hair Jr., J. F., Anderson, R. E., Tatham, R. L., \& Black, W. C. (2009). Análise multivariada de dados. (6. ed.). São Paulo: Bookman.

Hart, O., Shleifer, A., \& Vishny, R. W. (1997, November). The proper scope of government: Theory and an application to prisons. The Quarterly Journal of Economics, 112 (4), 1127-61.

Lewis, M. (2006, January). Governance and corruption in public health care systems. [S.l.]: Center for Global Development, Working Paper n. 78

Matias-Pereira, J. (2002, Outubro). Reforma do Estado e transparência: estratégias de controle da corrupção no Brasil. Anais do Congreso Internacional del CLAD sobre la Reforma del Estado y de La Administración Pública, Lisboa, Portugal, 7.

Matias-Pereira, J. (2005, Abril-Junho). Reforma do Estado e controle da corrupção no Brasil. Cadernos de Pesquisa em Administração (USP), 12 (2), 1-17.

Mimicopoulos, M. G., Kyj, L., \& Sormani, N. (2007). Public governance indicators: a literature review. New York: ST/ESA/PAD/SER.E/100, United Nations, Department of Economic and Social Affairs.

Motta, A. R. (2010). O combate ao desperdício no gasto público: uma reflexão baseada na comparação entre os sistemas de compra privado, público federal norte-americano e brasileiro. Dissertação de mestrado, Universidade Estadual de Campinas, Instituto de Economia, Campinas, SP, Brasil.

Niyama, J. K., \& Silva, C. A. T. (2002, Maio-Agosto). Teoria da contabilidade. (2. ed.). São Paulo: Atlas, 2011.

Oliveira, M. C. (2002). Análise dos periódicos brasileiros de contabilidade. Revista Contabilidade e Finanças - USP, São Paulo, 13 (29), 68-86.

Peixoto, S. G. D., Rocha, F. F., Nishijima, M., \& Pstali, F. A. S. (2012). Decentralization and corruption: evidence from primary health-care programmes. Applied Economics Letters, 19 (18), 1885-1888.

Portal Brasil. (2009, Dezembro). O que é o SUS. Brasília. Recuperado em 3 julho, 2012, de http://www.brasil.gov.br/sobre/saude/atendimento/o-que-e-sus.

Portal Brasil. (2011). Diretrizes do Governo. Recuperado em 3 julho, 2012, de http://www2.planalto.gov.br/presidenta/diretrizes-de-governo.

Reinikka, R., \& Svensson, J. (2004). The power of information: evidence from a newspaper campaign to reduce capture. [S.1.]: Working Paper, IIES, Stockholm University.

Reinikka, R., \& Svensson, J. (2005, April-May). Fighting corruption to improve schooling: evidence from a newspaper campaign in Uganda. Journal of the European Economic Association, Papers and Proceedings of the Nineteenth Annual Congress of the European Economic Association, 3 (2/3), 259-267.

Reis, L. G. dos, Slomski, V., Pereira, C. A., \& Mello, G. R. de. (2007, Setembro). A pesquisa brasileira em contabilidade do setor público: uma análise após a implementação da Lei de Responsabilidade Fiscal. RIC/UFPE - Revista de Informação Contábil, 1 (1), 89-101.

Resolução CFC n. 1209, de 27 de novembro de 2009 (2009). Aprova a NBC TA 260, Comunicação com os Responsáveis pela Governança. Conselho Federal de Contabilidade - CFC. Recuperado em 4 setembro, 2013, de http://www.cfc.org.br/sisweb/sre/detalhes_sre. aspx?Codigo $=2009 / 001209$.

Rosa, L. (2011, Abril). Depois da casa própria, o maior sonho do brasileiro é dar plano de saúde à família. Clipping de Notícias Abramge, Brasil Econômico, Especial Seguros.

Sodré, A. C. de A., \& Alves, M. F. C. (2010). Relação entre emendas parlamentares e corrupção municipal no Brasil: estudo dos relatórios do programa de fiscalização da Controladoria-Geral da União. Revista Administração Contemporânea [online], 14 (3), 414-433. 


\section{ANEXO}

Municípios que receberam fiscalização especial da CGU através do Programa de Sorteio dos Municípios

\section{SORTEIO PÚBLICO DE MUNICÍPIOS}

\begin{tabular}{|c|c|c|c|c|c|}
\hline \multicolumn{3}{|c|}{$31^{\circ}$} & \multicolumn{3}{|c|}{$32^{\circ}$} \\
\hline Ordem sorteio & Município & UF & Ordem sorteio & Município & UF \\
\hline $1^{\circ}$ & Mauá da Serra & $\mathrm{PR}$ & $1^{\circ}$ & Normandia & $\mathrm{RR}$ \\
\hline $2^{\circ}$ & Wenceslau Braz & PR & $2^{\circ}$ & Douradina & MS \\
\hline $3^{\circ}$ & Guaporema & PR & $3^{\circ}$ & São Félix do Araguaia & MT \\
\hline $4^{\circ}$ & São Gabriel & $\mathrm{BA}$ & $4^{\circ}$ & Xambioá & TO \\
\hline $5^{\circ}$ & Itaparica & $\mathrm{BA}$ & $5^{\circ}$ & Nossa Senhora das Dores & SE \\
\hline $6^{\circ}$ & Palmas de Monte Alto & BA & $6^{\circ}$ & Cerejeiras & $\mathrm{RO}$ \\
\hline $7^{\circ}$ & Pojuca & $\mathrm{BA}$ & $7^{\circ}$ & Cardoso Moreira & RJ \\
\hline $8^{\circ}$ & Cachoeira & $\mathrm{BA}$ & $8^{\circ}$ & Anajás & PA \\
\hline $9^{\circ}$ & Arroio do Meio & RS & $9^{\circ}$ & Santa Filomena & $\mathrm{PE}$ \\
\hline $10^{\circ}$ & Doutor Ricardo & RS & $10^{\circ}$ & Pedro Velho & $\mathrm{RN}$ \\
\hline $11^{\circ}$ & Pouso Novo & RS & $11^{\circ}$ & Umirim & CE \\
\hline $12^{\circ}$ & Pedro Osório & RS & $12^{\circ}$ & Luziânia & GO \\
\hline $13^{\circ}$ & Jeriquara & $\mathrm{SP}$ & $13^{\circ}$ & Senador La Rocque & MA \\
\hline $14^{\circ}$ & Ipuã & $\mathrm{SP}$ & $14^{\circ}$ & Imaculada & PB \\
\hline $15^{\circ}$ & Viradouro & $\mathrm{SP}$ & $15^{\circ}$ & São João da Serra & $\mathrm{PI}$ \\
\hline $16^{\circ}$ & Dracena & SP & $16^{\circ}$ & Peritiba & SC \\
\hline $17^{\circ}$ & Poloni & $\mathrm{SP}$ & $17^{\circ}$ & Doutor Pedrinho & SC \\
\hline $18^{\circ}$ & Coronel Xavier Chaves & MG & $18^{\circ}$ & Santo Inácio & PR \\
\hline $19^{\circ}$ & Capim Branco & MG & $19^{\circ}$ & Inajá & PR \\
\hline $20^{\circ}$ & São João da Lagoa & MG & $20^{\circ}$ & Heliópolis & $\mathrm{BA}$ \\
\hline $21^{\circ}$ & Carvalhos & MG & $21^{\circ}$ & luiú & $\mathrm{BA}$ \\
\hline $22^{\circ}$ & Santo Antônio do Monte & MG & $22^{\circ}$ & Erval Seco & RS \\
\hline $23^{\circ}$ & Matias Barbosa & MG & $23^{\circ}$ & Caseiros & RS \\
\hline $24^{\circ}$ & Frei Inocêncio & MG & $24^{\circ}$ & Votorantim & SP \\
\hline $25^{\circ}$ & Amajari & $\mathrm{RR}$ & $25^{\circ}$ & Ribeirão Branco & SP \\
\hline $26^{\circ}$ & Primavera de Rondônia & $\mathrm{RO}$ & $26^{\circ}$ & Bariri & SP \\
\hline $27^{\circ}$ & Tefé & $\mathrm{AM}$ & $27^{\circ}$ & Planura & MG \\
\hline $28^{\circ}$ & Frei Paulo & SE & $28^{\circ}$ & Caxambu & MG \\
\hline $29^{\circ}$ & Venda Nova do Imigrante & ES & $29^{\circ}$ & Senador Cortes & MG \\
\hline
\end{tabular}


continuação

\begin{tabular}{|c|c|c|c|c|c|}
\hline \multicolumn{3}{|c|}{$31^{\circ}$} & \multicolumn{3}{|c|}{$32^{\circ}$} \\
\hline Ordem sorteio & Município & UF & Ordem sorteio & Município & UF \\
\hline $30^{\circ}$ & Bonito & MS & $30^{\circ}$ & Campestre & MG \\
\hline $31^{\circ}$ & Taquarana & $\mathrm{AL}$ & $31^{\circ}$ & Pedra Branca do Amapari & AP \\
\hline $32^{\circ}$ & Girau do Ponciano & $\mathrm{AL}$ & $32^{\circ}$ & Maracaju & MS \\
\hline $33^{\circ}$ & Cabo Frio & RJ & $33^{\circ}$ & Tabatinga & AM \\
\hline $34^{\circ}$ & Inhangapi & $\mathrm{PA}$ & $34^{\circ}$ & Curralinho & PA \\
\hline $35^{\circ}$ & São Sebastião da Boa Vista & $\mathrm{PA}$ & $35^{\circ}$ & Nova Maringá & MT \\
\hline $36^{\circ}$ & Itaituba & $\mathrm{PA}$ & $36^{\circ}$ & São Bento do Tocantins & TO \\
\hline $37^{\circ}$ & Santa Rosa do Tocantins & TO & $37^{\circ}$ & Pedro Canário & ES \\
\hline $38^{\circ}$ & Cocalinho & MT & $38^{\circ}$ & Pendências & $\mathrm{RN}$ \\
\hline $39^{\circ}$ & Jardim do Seridó & $\mathrm{RN}$ & $39^{\circ}$ & Branquinha & $\mathrm{AL}$ \\
\hline $40^{\circ}$ & Luís Gomes & $\mathrm{RN}$ & $40^{\circ}$ & Independência & $\mathrm{CE}$ \\
\hline $41^{\circ}$ & Lucrécia & $\mathrm{RN}$ & $41^{\circ}$ & Surubim & PE \\
\hline $42^{\circ}$ & Timbaúba & $\mathrm{PE}$ & $42^{\circ}$ & Penalva & MA \\
\hline $43^{\circ}$ & Lagoa do Carro & $\mathrm{PE}$ & $43^{\circ}$ & Alegrete do Piauí & $\mathrm{PI}$ \\
\hline $44^{\circ}$ & Camutanga & $\mathrm{PE}$ & $44^{\circ}$ & Riacho dos Cavalos & PB \\
\hline $45^{\circ}$ & Granja & CE & $45^{\circ}$ & Americano do Brasil & $\mathrm{GO}$ \\
\hline $46^{\circ}$ & Jucás & $\mathrm{CE}$ & $46^{\circ}$ & Capinzal & SC \\
\hline $47^{\circ}$ & Mucambo & $\mathrm{CE}$ & $47^{\circ}$ & Bom Jardim da Serra & SC \\
\hline $48^{\circ}$ & Arari & MA & $48^{\circ}$ & Congonhinhas & PR \\
\hline $49^{\circ}$ & Água Doce do Maranhão & MA & $49^{\circ}$ & Pinhalão & PR \\
\hline $50^{\circ}$ & Mata Roma & MA & $50^{\circ}$ & Uibaí & $\mathrm{BA}$ \\
\hline $51^{\circ}$ & Paes Landim & $\mathrm{PI}$ & $51^{\circ}$ & Arataca & BA \\
\hline $52^{\circ}$ & Dom Expedito Lopes & $\mathrm{PI}$ & $52^{\circ}$ & Toropi & RS \\
\hline $53^{\circ}$ & Flores do Piauí & $\mathrm{PI}$ & $53^{\circ}$ & Alto Alegre & RS \\
\hline $54^{\circ}$ & Passagem & PB & $54^{\circ}$ & Pedregulho & SP \\
\hline $55^{\circ}$ & Natuba & $\mathrm{PB}$ & $55^{\circ}$ & Vargem & SP \\
\hline $56^{\circ}$ & Caldas Brandão & $\mathrm{PB}$ & $56^{\circ}$ & São João de Iracema & SP \\
\hline $57^{\circ}$ & Carmo do Rio Verde & $\mathrm{GO}$ & $57^{\circ}$ & São Sebastião do Rio Verde & MG \\
\hline $58^{\circ}$ & Novo Gama & GO & $58^{\circ}$ & Abadia dos Dourados & MG \\
\hline $59^{\circ}$ & Piratuba & $\mathrm{SC}$ & $59^{\circ}$ & Felisburgo & MG \\
\hline $60^{\circ}$ & Caçador & SC & $60^{\circ}$ & Itamogi & MG \\
\hline
\end{tabular}




\section{APÊNDICE 1}

Visando facilitar a compreensão de como foi construído o banco de dados, a seguir apresentamos alguns exemplos de dados extraídos dos Relatórios dos Auditores do Programa de Fiscalização por Sorteios Públicos da CGU e classificados de acordo com as variáveis de desperdício ativo e passivo presentes na Tabela 1.

Superfaturamento: "Medicamentos básicos adquiridos por preços superiores aos praticados no mercado."

Licitação com desvio de recursos: "Execução a menor do objeto, em comparação com as despesas realizadas, com prejuízo de pelo menos R $\$ 24.026,67$."

Inexistência ou fraca atuação do conselho: "Ausência de condições mínimas para o funcionamento do Conselho Municipal de Saúde de Bom Jardim da Serra-SC, não comprovação do repasse de informações ao Conselho e baixa periodicidade em suas reuniões."

Licitação com falta de concorrência: "Empresa selecionada em convite sem o requisito de o mínimo de três propostas válidas."

Não ocorreu a contrapartida: "Descumprimento pelo Estado e pelo Município dos valores pactuados para distribuição de medicamentos da Farmácia Básica à população do município."

Não pagamento de tributo: "Não comprovação do recolhimento das contribuições previdenciárias."

Obra inacabada: "Obras paralisadas e inacabadas, relativas ao Convênio n ${ }^{\circ}$ EP 1445/2005, devido ao abandono das mesmas pela empresa contratada, inviabilizando o atingimento total dos objetivos pretendidos e ferindo os princípios constitucionais da eficiência e da economicidade." 